\title{
Dinâmica de Transmissão das Moléstias Infecciosas: Múltiplas Infecções e Período de Latência ${ }^{1}$
}

S.M. RAIMUNDO ${ }^{2}$, Faculdade de Medicina da Universidade de São Paulo, FMUSP, Av. Dr. Arnaldo 455, 01246-903 São Paulo, SP, Brasil

H.M. YANG ${ }^{3}$, Departamento de Matemática Aplicada, Instituto de Matemática, Estatística e Computação Científica, UNICAMP, Cx.P. 6065, 13081-970 Campinas, SP, Brasil.

\begin{abstract}
Resumo. Neste trabalho propõe-se um modelo matemático do tipo SEIRE, com população constante, considerando a reinfecção dos indivíduos. Diferente da maioria dos modelos clássicos em epidemiologia, e principalmente por causa da hipótese de múltiplas infecções, o modelo apresenta uma bifurcação "backward", o que pode resultar em um comportamento que não é o usual de um modelo baseado na lei da ação das massas. Ou seja, quando o número de reprodutibilidade basal, $\mathcal{R}_{0}$, esteja entre um valor crítico e um, podem surgir dois equilíbrios endêmicos além do equilíbrio trivial. Procura-se explorar o efeito das múltiplas infecções em conjunção com o período de latência, mostrando a importância relativa entre eles. De um modo geral, quando a permanência dos indivíduos no estágio latente for muito lenta, como é o caso da tuberculose, as infecções múltiplas têm importância não só na prevalência, mas tembém na questão da erradicação da doença.
\end{abstract}

\section{Introdução}

Atualmente, um dos interesses da teoria epidemiológica é a existência de múltiplos equilíbrios endêmicos e os mecanismos epidemiológicos que os produzem. Os modelos matemáticos que consideram múltiplos equilíbrios endêmicos apresentam o fenômeno de bifurcação que, em geral, está associado a um conjunto de valores limiares de parâmetros no qual o equilíbrio do sistema considerado muda a sua estabilidade.

O valor limiar mais conhecido e usado em epidemiologia é o número de reprodutibilidade basal da doença, $\mathcal{R}_{0}$, definido como o número médio de infecções secundárias produzidas por um único indivíduo infeccioso, numa população inteiramente suscetível, na ausência de qualquer heterogeneidade e, também, de múltiplas infecções. Em sua maioria, os modelos clássicos (SIS, SIRS ou SEIRS) com taxa de transmissão e população constantes, apresentam um único equilíbrio endêmico

\footnotetext{
${ }^{1}$ Apoio financeiro CNPq (Edital Universal 01/2002)

${ }^{2}$ silviamr@dim.fm.usp.br

${ }^{3}$ hyunyang@ime.unicamp.br
} 
estável. Assim, se $\mathcal{R}_{0}<1$, o equilíbrio livre da doença é globalmente assintoticamente estável (G.A.E.) e a doença não se estabelece na população. Porém, para $\mathcal{R}_{0}>1$, o equilíbrio endêmico é G.A.E., ou seja, a fração de indivíduos infecciosos cresce e a doença tende para seu nível endêmico. Assim, a característica destes modelos fundamentados na lei da ação das massas é a presença de bifurcação "forward".

Quando, porém, associa-se ao modelo a possibilidade de múltiplas infecções, então pode exibir comportamentos mais complexos como, por exemplo, a bifurcação "backward". Uma bifurcação deste tipo está intimamente relacionada ao fato do indivíduo infectado estar sujeito a múltiplas infecções durante o período de incubação. Sob o ponto de vista matemático, isto significa a existência do equilíbrio trivial e de um equilíbrio endêmico estável e de um equilíbrio endêmico instável, em situação especial em que o número de reprodutibilidade basal é menor que unidade, $\mathcal{R}_{0}<1$.

Portanto, a presença de bifurcação "backward" em modelos epidemiológicos tem implicações importantes, pois pequenas mudanças nos valores dos parâmetros do modelo podem produzir mudanças significativas na dinâmica do sistema. Por exemplo, imagine uma população livre da doença, com o valor de $\mathcal{R}_{0}$ variando lentamente. Na bifurcação "forward", quando $\mathcal{R}_{0}$ ultrapassa o valor um, a doença consegue se estabelecer na população em um nível endêmico, que cresce com aumento de $\mathcal{R}_{0}$, de uma situação outrora livre da doença. Por outro lado, na bifurcação "backward" quando $\mathcal{R}_{0}$ ultrapassa o valor um, a doença pode-se estabelecer na população em um nível endêmico relativamente alto. Contudo, neste caso, a endemicidade da doença pode não surgir de uma situação previamente ausente da doença, pois mesmo para valores de $\mathcal{R}_{0}$ entre um valor crítico $\left(\mathcal{R}_{p}\right)$ e um, pode ser que a doença seja endêmica na comunidade. Dessa forma, uma característica da presença de múltiplas infecções é a possibilidade de, mesmo para $\mathcal{R}_{0}<1$, existir um equilíbrio endêmico estável. Assim, para que o equilíbrio trivial seja estável independentemente de qualquer outra condição, não basta baixar $\mathcal{R}_{0}$ para valores menores que um, mas sim, para um valor abaixo do limiar $\mathcal{R}_{p}$, que é menor que um. Ressalta-se, porém, que existe uma outra alternativa para erradicar a doença para $\mathcal{R}_{p}<\mathcal{R}_{0}<1$ : baixar $\mathcal{R}_{0}$ para valores menores que um, e, então, baixar a incidência para domínio de atração do equilíbrio trivial.

Neste trabalho desenvolve-se um modelo matemático para a transmissão de moléstias infecciosas com o propósito de avaliar o impacto das infecções múltiplas e do período de incubação na sua dinâmica. Por apresentar uma epidemiologia diferente da maioria das doenças infecciosas, pois apresenta tempo extremamente variável entre a prima-infeç̧̃a com o Mycobacterium tuberculosis (MTB) e a doença, a Tuberculose (TB) é um exemplo de aplicação do modelo proposto. Na Seção 2, desenvolve-se o modelo matemático do tipo SEIRE, com população constante e múltiplas infecções. Na Seção 3, apresenta-se um estudo da estabilidade do equilíbrio livre da doença e dos equilíbrios endêmicos. Na Secção 4, apresenta-se discussão do modelo e as conclusões. 


\section{O Modelo}

Baseado na história natural da doença, uma população total, $N=S^{\prime}+E^{\prime}+I^{\prime}+R^{\prime}$, considerada constante, é dividida em quatro compartimentos, no instante de tempo $t$ : $S^{\prime}$, número de indivíduos suscetíveis; $E^{\prime}$, indivíduos expostos (infectados, porém não-infecciosos); $I^{\prime}$, indivíduos infecciosos e $R^{\prime}$, indivíduos parcialmente imunes, portanto sujeitos a reinfecções. Quando indivíduos suscetíveis são infectados por agentes causadores da doença, tornam-se indivíduos expostos. Após um período de tempo $\gamma^{-1}$, onde $\gamma$ é a taxa de incubação, estes indivíduos tornam-se infecciosos. Durante este período de incubação pode ocorrer outras infecções. Os indivíduos infecciosos, após período de tempo $\delta^{-1}$, onde $\delta$ é a taxa de recuperação, estes indivíduos tornam-se recuperados, porém não com imunidade perfeita, podendo, assim, ser reinfectados.

Para a dinâmica da transmissão da infecção, considera-se a "verdadeira" lei da ação das massas. Assim, a força de infecção é proporcional à densidade de indivíduos infecciosos $\left(I^{\prime} / N\right)$. Pode-se, assim, dividir indivíduos em cada compartimento pelo número total $N$, e usa-se as frações correspondentes a cada compartimento dadas por $S, E, I$ e $R$. O modelo de múltiplas infecções, com reinfecção, é descrito pelo seguinte sistema de equações diferencias ordinárias

$$
\left\{\begin{array}{l}
\frac{d S}{d t}=\mu+\alpha I-\beta S I-\mu S \\
\frac{d E}{d t}=\beta S I+q \beta R I-p \beta E I-\gamma E-\mu E \\
\frac{d I}{d t}=p \beta E I+\gamma E-(\mu+\alpha+\delta) I \\
\frac{d R}{d t}=\delta I-q \beta R I-\mu R
\end{array}\right.
$$

onde $\frac{d N}{d t}=0$, pois $S+E+I+R=1$ (população constante) é devido à existência de um fluxo de entradas constante $(\mu+\alpha I)$ na classe dos suscetíveis, $S$. A dinâmica vital inclui a taxa de mortalidade natural, $\mu$, e a taxa de morte adicional pela doença, $\alpha$. Os indivíduos suscetíveis tornam-se infectados através do contacto com os indivíduos infecciosos de acordo com a lei da ação das massas, sendo $\beta$ definido como o coeficiente de transmissão. Uma vez infectados, esses indivíduos podem permanecer no estado exposto, $E$, até completar o período de incubação, ou 'seguir um atalho' por uma nova infecção e tornar-se infeccioso (desenvolver a doença). Esta nova infecção (denominada de múltipla infecção) ocorre conforme $p \beta$, onde o parâmetro $p$, com $0 \leq p \leq 1$, representa o nível de força de múltipla infecção. Como os indivíduos recuperados podem-se reinfectar, define-se o coeficiente de transmissão por $q \beta$, onde o parâmetro $q$, com $0 \leq q \leq 1$, representa o nível de proteção parcial (se $q=0$, tem-se proteção perfeita por uma imunidade perene). Os parâmetros $\gamma \mathrm{e}$ $\delta$ foram previamente definidos. Todos os parâmetros do modelo são positivos.

\section{Análise do Modelo}

Apresenta-se a análise do sistema de equações (2.1), determinando os pontos de equilíbrio e estudando a estabilidade destes pontos, em duas partes: trivial e nãotrivial. 


\subsection{Equilíbrio livre da doença}

O equilíbrio trivial do sistema de equações (2.1), ou a população livre da doença, é dado por $\bar{S}=1$ e $\bar{E}=\bar{I}=\bar{R}=0$, ou $P^{0}=(1,0,0,0)$. A estabilidade de $P^{0}$ é determinada pelos autovalores correspondentes à matriz jacobiana associada ao sistema de equações (2.1) calculada em $P^{0}$. Dois autovalores são obtidos facilmente, dados por $\lambda_{1}=\lambda_{2}=-\mu$. Os outros dois, designados por $\lambda_{3}$ e $\lambda_{4}$, são dados pelas raízes do seguinte polinômio característico

$$
\Lambda(\lambda)=\lambda^{2}+a_{1} \lambda+a_{2}=0,
$$

com $a_{1}=2 \mu+\alpha+\gamma+\delta$ e $a_{2}=(\mu+\gamma)(\mu+\alpha+\delta)\left(1-\mathcal{R}_{0}\right)$, onde o número de reprodutibilidade basal é definido como

$$
\mathcal{R}_{0}=\frac{\beta}{\beta_{0}}
$$

onde

$$
\beta_{0}=\frac{(\mu+\gamma)(\mu+\alpha+\delta)}{\gamma}
$$

é o valor limiar para $\beta$.

Para verificar a estabilidade de $P^{0}$, deve mostrar que os autovalores associados ao polinômio (3.1) são negativos (se reais) ou terem parte real negativa (se complexos). Como $a_{1}>0$, então para que todos os autovalores sejam negativos basta ter $a_{2}>0$, isto é, $1-\mathcal{R}_{0}>0$, o que é verdade se e somente se $\mathcal{R}_{0}<1$. Dessa forma, sempre que $\mathcal{R}_{0}<1$ (ou $\beta<\beta_{0}$ ), o equilíbrio livre da doença, $P^{0}=(1,0,0,0)$, é localmente assintoticamente estável (L.A.E.); caso contrário, é instável (L.A.I.). Observe que o valor limiar $\mathcal{R}_{0}$ não depende dos valores dos níveis de múltipla infecção e reinfecção (resumidos, respectivamente, pelos parâmetros $p$ e $q$ ), como era de se esperar.

\subsection{Natureza e quantidade de equilíbrios endêmicos}

O ponto de equilíbrio não-trivial, $P^{*}=\left(S^{*}, E^{*}, I^{*}, R^{*}\right)$ do sistema e equações (2.1), é dado pelas coordenadas

$$
\left\{\begin{aligned}
S^{*} & =\frac{\mu+\alpha I^{*}}{\mu+\beta I^{*}} \\
E^{*} & =\frac{(\mu+\alpha+\delta)}{\gamma+p \beta I^{*}} I^{*} \\
R^{*} & =\frac{\delta}{\mu+q \beta I^{*}} I^{*},
\end{aligned}\right.
$$

onde o valor de $I^{*}$ não-trivial é obtido como raízes da equação de terceiro grau

$$
a_{3}^{*} x^{3}+a_{2}^{*} x^{2}+a_{1}^{*} x+a_{0}^{*}=0,
$$

$\operatorname{com} x=\beta I^{*}$, onde $x$ tem dimensão $[\text { tempo }]^{-1}$ e os coeficientes são dados por

$$
\left\{\begin{aligned}
a_{3}^{*}= & p q \\
a_{2}^{*}= & p(\mu+\delta)+q(\mu+\alpha+\delta+\gamma)+p q[\beta-(\mu+\alpha)] \\
a_{1}^{*}= & (\mu+\gamma)(\mu+\alpha+\delta)\left(1-\frac{\alpha}{\beta_{0}}\right)+p \mu[(\mu+\alpha+\delta)-\beta] \\
& +q(\mu+\gamma)(\mu+\alpha+\delta)\left(1-\mathcal{R}_{0}-\frac{\delta}{\beta_{0}}\right) \\
a_{0}^{*}= & \mu(\mu+\gamma)(\mu+\alpha+\delta)\left(1-\mathcal{R}_{0}\right) .
\end{aligned}\right.
$$


Os parâmetros $\mathcal{R}_{0}$ e $\beta_{0}$ são dados pelas equaçãos (3.2) e (3.3), respectivamente. Note que $I^{*}=0$ é uma solução do sistema de equações (2.1), o equilíbrio trivial $P^{0}$.

Pode-se mostrar que na ausência de múltiplas infecções, para $p=0$, ocorre a bifurcação "forward", isto é, se $\mathcal{R}_{0}<1$, o equilíbrio trivial $P^{0}$ é G.A.E. e a doença é eliminada da população. Se $\mathcal{R}_{0}>1$, o equilíbrio trivial $P^{0}$ torna-se instável, e o equilíbrio endêmico, que é único e é dado pela solução da equação (3.5), é G.A.E., ou seja, a doença é prevalente na população. Este comportamento dinâmico, que independe do nível de reinfecção dado pelo parâmetro $q$, corresponde ao modelo clássico fundamentado na lei da ação das massas. Assim, sem perda de generalidades, pode-se assumir $q=1$, porém $0<p \leq 1$, pois neste caso ocorrem comportamentos dinâmicos não associados à lei da ação das massas, como a existência de dois equilíbrios não-triviais positivos e a bifurcação "backward".

Primeiramente, sendo $x=-\mu$ uma raiz para o polinômio (3.5) para $q=1$, então $I^{*}=-\frac{\mu}{\beta}$ é uma raiz real negativa do sistema e equações (2.1). Resta determinar quais são as condições de existência para que outras duas raízes sejam reais e positivas. Observe que o equilíbrio não-trivial positivo existe para o sistema de equações (2.1), quando existir solução $x$ positiva para o polinômio (3.5). Lembre que $q=1$ representa o caso em que todos os indivíduos recuperados podem-se reinfectar a uma taxa idêntica a dos suscetíveis, mesmo que o sistema imune já tenha sido estimulado por infecções precedentes, portanto, é um modelo tipo SEIR, ou seja, a imunidade é temporária.

Fatorando a raiz negativa $x=-\mu$, o polinômio (3.5) fica reduzido a um polinômio de grau dois, dado por

$$
\chi(x)=a_{2} x^{2}+a_{1} x+a_{0}=0,
$$

onde os coeficientes são dados por

$$
\left\{\begin{array}{l}
a_{2}=p \\
a_{1}=[(\mu+\alpha+\delta+\gamma)+p(\mu+\alpha+\delta)]\left(1-\frac{\beta}{\beta_{1}}\right) \\
a_{0}=(\mu+\gamma)(\mu+\alpha+\delta)\left(1-\mathcal{R}_{0}\right),
\end{array}\right.
$$

com o valor $\beta_{1}$, com $p \neq 0$, sendo dado por

$$
\beta_{1}=\frac{(\mu+\alpha+\delta+\gamma)}{p}+(\mu+\alpha+\delta)
$$

A dependência de $a_{1}$ com $\beta_{1}$ faz surgir novos comportamentos dinâmicos.

Note que, para $p=0, a_{1}=(\mu+\alpha+\delta+\gamma)>0$ e tem-se resultados do modelo clássico da lei da ação das massas. O mesmo ocorre quando $\beta_{0}<\beta_{1}$, dados pelas equações (3.3) e (3.9), respectivamente. Porém, quando $\beta_{0}>\beta_{1}$, verifica-se que, para certos valores do coeficiente de transmissão $\beta<\beta_{0}$, justamente para $\mathcal{R}_{0}<$ 1 , pode ocorrer múltiplos equilíbrios endêmicos devido à múltipla infecção. Para elucidar este fenômeno, determina-se a posição relativa entre $\beta_{0}$ e $\beta_{1}$, definindo a função $f(p)$ como

$$
f(p) \equiv \beta_{1}-\beta_{0}=\gamma(\mu+\alpha+\delta+\gamma)\left(1-\frac{p}{p_{0}}\right),
$$


onde deve-se ter $0<p \leq 1$ e $p_{0}$ é dado por

$$
p_{0}=\frac{\mu(\mu+\alpha+\delta)}{\gamma(\mu+\alpha+\delta+\gamma)}
$$

com $0 \leq p_{0} \leq 1$

A única possibilidade de existir até duas raízes reais positivas para o polinômio (3.7) é quando seus coeficientes, dada pela equação (3.8), satisfizerem $a_{1}<0 \mathrm{e}$ $a_{0}>0$, uma vez que $a_{2}>0$. Estas condições são satisfeitas quando $\beta_{0}>\beta_{1}$, isto é, quando $f(p)<0$, o que ocorre para

$$
p>p_{0} .
$$

Porém, deve-se ter, da equação (3.10), $p_{0}>0$ (naturalmente satisfeita) e $p_{0}<1$ (como $p>p_{0}, p_{0}$ não pode ser igual a um), que é satisfeita se

$$
g(\gamma) \equiv \gamma^{2}+(\mu+\alpha+\delta) \gamma-\mu(\mu+\alpha+\delta)<0 .
$$

O polinômio $g(\gamma)$ tem duas raízes reais positiva e negativa, dadas, respectivamente, por $\gamma_{+}$e $\gamma_{-}$. Logo, $g(\gamma)<0$ se e somente se $\gamma<\gamma_{+}$, onde

$$
\gamma_{+}=\frac{\mu+\alpha+\delta}{2}\left(\sqrt{1+\frac{4 \mu}{\mu+\alpha+\delta}}-1\right) .
$$

Note que $g(\gamma=\mu)=\mu^{2}>0, \log \mu>\gamma_{+}$, mostrando que $\gamma_{+}$é um valor muito pequeno.

Portanto, para $\beta_{0}>\beta_{1}$ e $\gamma<\gamma_{+}$o polinômio (3.7) pode ter até duas raízes positivas apenas no intervalo $\beta_{1}<\beta<\beta_{0}$. No entanto, pode-se mostrar que para $\beta_{c}<\beta<\beta_{0}$, com $\beta_{1}<\beta_{c}$, tem-se de fato duas raízes reais positivas, com $\beta_{c}$ dado por

$$
\beta_{c}=\left(\beta_{1}-\frac{2 \gamma}{p}\right)+\frac{2 \gamma}{p} \sqrt{1+\frac{p}{\gamma}\left(\beta_{0}-\beta_{1}\right)},
$$

sendo que em $\beta=\beta_{c}$, tem-se raiz real positiva dupla. As raízes reais postivas são designadas por $I_{+}^{*}$ e $I_{-}^{*}$, que são, respectivamente, a maior e a menor das soluções. Contudo, para $\beta<\beta_{c}$ não há solução real postiva, e para $\beta>\beta_{0}$, uma única solução real positiva. Pode-se dividir as desigualdades $\beta_{c}<\beta<\beta_{0}$ por $\beta_{0}$, e escrever como

$$
\frac{\beta_{c}}{\beta_{0}} \equiv \mathcal{R}_{p}<\mathcal{R}_{0}<1
$$

mostrando, assim, a possibilidade de endemia para $\mathcal{R}_{0}<1$.

Para analisar a estabilidade dos pontos de equilíbrio, sabe-se que para $q=1 \mathrm{o}$ modelo é do tipo SEIRS. Portanto, pode-se usar $Y=S+R$ no sistema dinâmico (2.1) e utilizar o valor em equilíbrio dado por

$$
Y^{*}=S^{*}+R^{*}=\frac{\mu+(\delta+\alpha) I^{*}}{\mu+\beta I^{*}},
$$


da equação (3.4). Este procedimento reduz em uma dimensão o sistema dinâmico, resultando em variáveis dinâmicas $(Y, E, I)$. Neste novo sistema dinâmico, os autovalores são as raízes do polinômio característico de terceiro grau dado por

$$
\Lambda(\lambda)=\lambda^{3}+b_{2} \lambda^{2}+b_{1} \lambda+b_{0},
$$

com coeficientes dados por

$$
\left\{\begin{array}{l}
b_{2}=2 \mu+(p+1) \beta I^{*}+\gamma\left(1+\frac{E^{*}}{I^{*}}\right) \\
b_{1}=\left[\left(\gamma+p \beta I^{*}\right)+\left(\mu+\beta I^{*}\right)+\gamma \frac{E^{*}}{I^{*}}\right]+\frac{b_{0}}{\mu} \\
b_{0}=\mu \beta\left[\left(\gamma+p \beta I^{*}\right)^{2}+(\gamma-p \mu)(\mu+\alpha+\delta)\right] \frac{I^{*}}{\gamma+p \beta I^{*}} .
\end{array}\right.
$$

A análise será feita de acordo com o valor de $\gamma$ relativo ao valor de $p \mu$, para $\beta_{0}>\beta_{1}$. Recorde que para $\beta_{0}<\beta_{1}$, recupera-se os resultados clássicos, ou seja, o único equilíbrio não-trivial é estável para $\mathcal{R}_{0}>1$, e instável caso contrário.

Primeiro, pode-se mostrar que $\gamma>p \mu>p_{0} \mu>\gamma_{+}$, ou seja, se $\gamma>p \mu$ então $\gamma>\gamma_{+}$, e o polinômio (3.7) tem uma única solução real positiva somente para $\beta>\beta_{0}$. Assim, se $\gamma>p \mu$, então $b_{0}>0$ implica que todos os outros coeficientes são positivos, e, de acordo com a conjectura em [1], o único equilíbrio não-trivial é L.A.E. Para $\beta<\beta_{0}$, só existe o equilíbrio trivial, que é G.A.E.

Para $\gamma<p \mu$, note que se $b_{0}>0$, então todos os outros coeficientes serão positivos. A estabilidade dos pontos de equilíbrio é procedida de seguinte modo. Primeiro, calcula-se um $I^{*}=z>0$ tal que $b_{0}=0$, ou seja, $Z(z)=\left(\gamma+p \beta I^{*}\right)^{2}-$ $(p \mu-\gamma)(\mu+\alpha+\delta)=0$. Pode-se mostrar que, para que se tenha uma raiz positiva para $z$, designada por $z_{+}$, a condição deve satisfazer $\gamma<\zeta(p) \leq \gamma_{+}$, onde

$$
\zeta(p)=\frac{\mu+\alpha+\delta}{2}\left(\sqrt{1+\frac{4 p \mu}{\mu+\alpha+\delta}}-1\right),
$$

$\operatorname{com} \zeta(1)=\gamma_{+}$. Note que se $Z(z)>0$, então $z>z_{+}$, e para $Z(z)<0, z<z_{+}$. Portanto, tem-se $b_{0}>0 \Leftrightarrow I^{*}>z_{+}$, e $b_{0}<0 \Leftrightarrow I^{*}<z_{+}$. Agora, calcula-se o valor da função $\chi\left(\beta z_{+}\right)$, dada pela equação (3.7). Pode-se mostrar que $\chi\left(\beta z_{+}\right)<0$ para $\gamma<\gamma_{+}$, sob as restrições $p_{0}<p<1$ e $\beta_{1}<\beta<\beta_{0}$, o que implica em $I_{-}^{*}<z_{+}<I_{+}^{*}$. Logo, tem-se que $b_{0}<0$ para $I_{-}^{*}<z_{+}$, mostrando que o menor equilíbrio não-trivial $I_{-}^{*}$ é L.A.I., pois não satisfaz uma das condições de Routh-Hurwitz; porém, para $I_{+}^{*}>z_{+}$tem-se que $b_{0}>0$, sendo a maior raiz $I_{+}^{*}$ L.A.E., conforme a conjectura em [1]. Contudo, neste caso, é fácil mostrar que a última condição de Routh-Hurwitz, dada por $b_{2} b_{1}-b_{0}>0$, é satisfeita pois

$$
\begin{aligned}
b_{2} b_{1}-b_{0}= & \mu\left[2 \mu+(p+1) \beta I^{*}+\gamma\left(1+\frac{E^{*}}{I^{*}}\right)\right]\left[\left(\gamma+p \beta I^{*}\right)+\left(\mu+\beta I^{*}\right)+\gamma \frac{E^{*}}{I^{*}}\right] \\
& +\left[\mu+(p+1) \beta I^{*}+\gamma\left(1+\frac{E^{*}}{I^{*}}\right)\right] \frac{b_{0}}{\mu}
\end{aligned}
$$

é positiva quando $b_{0}>0$.

Assim, tem-se que, para $\beta_{c}<\beta<\beta_{0}$, $I_{-}^{*}$ é instável e $I_{+}^{*}$ é estável (bifurcação "backward" para $\mathcal{R}_{p}<\mathcal{R}_{0}<1$ ), e para $\beta>\beta_{0}$, uma única raiz real positiva 
L.A.E. Mais ainda, a dinâmica da transmissão depende da condição inicial quando $\beta_{c}<\beta<\beta_{0}$, pois se $I(t=0)<I_{-}^{*}$, então a trajetória dinâmica dirige-se para o equilíbrio trivial; caso contrário, é atraída para $I_{+}^{*}$. Recorde que o equilíbrio trivial é estável para $\beta<\beta_{0}$, caso contrário, instável.

\section{Discussão e Conclusão}

O modelo tratou da questão de múltiplas infecções $(p \beta)$ e reinfecção $(q \beta)$, além da prima-infecção $(\beta)$. Mostrou-se que existe a possibilidade de bifurcação "backward" para $\mathcal{R}_{0}<1$, porém em condição muito especial: para $\gamma<\gamma_{+}<\mu$. Para $\gamma>\gamma_{+}$, a múltipla infecção não altera de modo algum a dinâmica qualitativa do modelo clássico que considera apenas a prima-infecção (com ou sem reinfecção). Assim, doenças que apresentem período de incubação $\left(\gamma^{-1}>\gamma_{+}^{-1}\right)$ muito extenso, maior que a vida média humana $\left(\mu^{-1}\right)$, o 'atalho' promovido por uma outra (múltipla) infecção para levar ao estado infeccioso (portanto, apresentando doença) é muito importante.

Para esclarecer, suponha $\gamma=0$ e $p>0$ (e $q=1$, sem perda de generalidade), situação que descreve uma dinâmica de transmissão em que muitas infecções são necessárias para tornar o indivíduo infeccioso, o que em geral é válido para infecções com macro-parasitas sexuadas. Pode-se mostrar que o equilíbrio trivial é L.A.E., existem sempre dois equilíbrios não-triviais $I_{-}^{*}$ (L.A.I.) e $I_{+}^{*}$ (L.A.E.) e, da equação (3.12), tem-se que $\beta_{c}=\beta_{1}$. Assim, para $\beta<\beta_{1}$, a doença é extinta na comunidade. Porém, para $\beta>\beta_{1}$, para introduções iniciais baixas de indivíduos infecciosos $(I(t=$ $\left.0)<I_{-}^{*}\right)$ a doença não consegue se manter, o que não é verdade para introduções elevadas $\left(I(t=0)>I_{-}^{*}\right)$ em que a endemicidade se estabelece em níveis de $I_{+}^{*}$, o que é muito comum na dinâmica de macro-parasitas sexuados. Pois, a probabilidade de acasalamento é fortemente dependente da carga parasitária, sendo muito reduzida para baixas cargas parasitárias [2]. Porém, se $p=0$ e $\gamma>0$, então tem-se a regra de $\mathcal{R}_{0}$ na dinâmica, ou seja, o modelo clássico da lei da ação das massas. Figura 1 mostra estas duas dinâmicas (curvas fina e tracejada, respectivamente, para múltiplas infecções e período de incubação, isoladamente).

Figura 1 mostra, também, o caso em que há uma composição de múltiplas infecções (no caso, uma segunda infeç̧ão) com período de incubação (curva grossa), quando $\gamma<\gamma_{+}$. Verifica-se que, mesmo para $\gamma$ muito pequeno, a dinâmica prevalente é a de passagem sem nova infecção para estado infeccioso, pois a estabilidade do equilíbrio trivial é ditada pela transição natural para estado infeccioso (ou, pelo parâmetro $\gamma$ ). Somente para baixas incidências ( $\beta$ pequeno), é que surge a dependência com condição inicial (ou, bifurcação "backward"), mostrando que a ocorrência de uma segunda (múltipla) infecção é um evento de busca ativa por indivíduos previamente infectados, o qual é dificultado para baixa prevalência. Assim, para incidências baixas $\left(\beta_{c}<\beta<\beta_{0}\right.$, claramente para $\left.\mathcal{R}_{p}<\mathcal{R}_{0}<1\right)$, a doença pode ser endêmica somente para uma introdução elevada de casos de infecção $\left(I(t=0)>I_{-}^{*}\right)$. Portanto, uma doença que apresente período longo de incubação e, ao mesmo tempo, em que uma segunda infecção acelere a manifestação da doença, pode ser descrito por este modelo. 


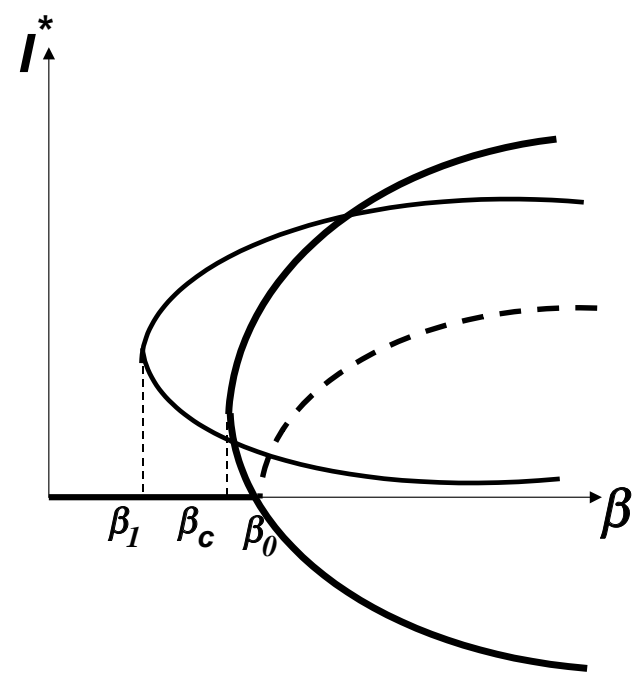

Figura 1: Diagrama de bifurcação para o modelo e para dois casos especiais.

Um exemplo de aplicação deste modelo é a $T B$, causada pela bactéria $M T B$, devido a seu longo período de incubação. Após a infecção com o $M T B$, conhecida como complexo primário ou prima-infecção, uma pequena fração dos indivíduos infectados desenvolvem rapidamente a TB. A probabilidade de evoluir para a doença em uma prima-infecção depende da virulência do bacilo, da fonte infectante e das características genéticas dos indivíduos infectados. Em geral, o complexo primário é bloqueado pelo sistema imunológico e a maioria dos indivíduos infectados permanecem no estado de latência durante um longo período de tempo ou por toda a vida. Porém TB pós-primária pode ocorrer no organismo que tem sua imunidade desenvolvida pelo complexo primário ou pela vacina. Uma pequena fração dos indivíduos primo-infectados evolui para a doença devido à reativação endógena (devido a $\gamma^{-1}$ ser muito extenso) ou então por receber uma nova carga bacilar (a reinfecção exógena, dado por $q$ ).

Embora TB seja uma doença grave, sendo que praticamente $100 \%$ dos casos sejam curados quando o tratamento é cumprido (dada pela taxa de recuperação $\delta)$, não confere imunidade perfeita ao paciente. Por outro lado, após o início do tratamento, é observada uma melhora muito rápida do paciente, que abandona o tratamento por ser muito agressivo e causar uma série de reações adversas, quando então o risco do paciente sofrer novas infecções é muito maior (a múltipla infecção, dado por $p$ ). Quando isto acontece, o paciente fica resistente às várias drogas usadas no combate a doença, caracterizando assim a $M D R-T B$ (tuberculose resistentes a 
múltiplas drogas). Assim, o paciente que abandona o tratamento não só aumenta seu risco às múltiplas infecções como também contribui com a nova epidemia de $T B$ pelas cepas resistentes às drogas.

Abstract. In this work a SEIRE mathematical model with constant population and reinfections is proposed. Although this model is based on the standard mass action law, our analysis shows that this model exhibit unusual behavior (backward bifurcation), due to the assumption of multiple infections. In other words, even when the reproductive number, $\mathcal{R}_{0}$, situates between a threshold value and unity, it is possible the occurrence of two endemic equilibria, besides the trivial equilibrium. We explore the relative importance of multiple infections and the periods of time that an individual spends in the latent class. Typically, when the natural history of infection presents a very long latent period, for instance tuberculosis, the multiple infections affect strongly on both prevalence and disease eradication efforts.

\section{Referências}

[1] M.B.F. Leite, R.C. Bassanezi e H.M. Yang, The Basic Reproduction Ratio for A Model of Directly Transmitted Infections Considering The Virus Charge and The Immunological Response, IMA J. Math. Appl. Med. Biol., 17, No. 1 (2000), 15-31.

[2] R.M. May, Togetherness amongst schistosome: Its effects on the dynamics of the infection, Math. Biosc., 35 (1977), 301-343.

[3] B.M. Murphy, B.H. Singer e D. Kirschner, On treatment of tuberculosis in heterogenous populations. J. Theoretical Biology, 223, No. 4 (2003), 391-404.

[4] S.M. Raimundo, H.M. Yang, R.C. Bassanezi e M.A.C. Ferreira, The Attraction Basins and the Assessment of the Transmission Coefficients for HIV and M. tuberculosis Infections among Women Inmates. J. Biol. Syst. 10, No. 1 (2002), 61-83.

[5] S.M. Raimundo, H.M. Yang, A.B. Engel e R.C. Bassanezi, An approach to estimating the Transmission Coefficients for AIDS and for Tuberculosis, Systems Analysis Modelling Simulation, 43, No. 4 (2003), 423-442.

[6] S.M. Raimundo e H.M. Yang, A mathematical model for exogenous reinfection and control of Tuberculosis transmission, Abstract Book: Computational and Mathematical Population Dynamics (CMPD), Trento, Italy, pg. 129, 2004.

[7] S.M. Raimundo e H.M. Yang, Tuberculose: questões sobre reinfecção exógena e reativação endógena, TEMA Tend. Mat. Apl. Comput., 6, No. 1 (2005), 121-130. 